PROCEEDINGS OF THE

AMERICAN MATHEMATICAL SOCIETY

Volume 136, Number 11, November 2008, Pages 3751-3757

S 0002-9939(08)09258-7

Article electronically published on June 20, 2008

\title{
THE STABILITY OF EXCEPTIONAL BUNDLES ON COMPLETE INTERSECTION 3-FOLDS
}

\author{
ROSA MARIA MIRÓ-ROIG AND HELENA SOARES \\ (Communicated by Ted Chinburg)
}

\begin{abstract}
A very long-standing problem in Algebraic Geometry is to determine the stability of exceptional vector bundles on smooth projective varieties. In this paper we address this problem and we prove that any exceptional vector bundle on a smooth complete intersection 3 -fold $Y \subset \mathbb{P}^{n}$ of type $\left(d_{1}, \ldots, d_{n-3}\right)$ with $d_{1}+\cdots+d_{n-3} \leq n$ and $n \geq 4$ is stable.
\end{abstract}

\section{INTRODUCTION}

Let $Y$ be a smooth projective variety over an algebraically closed field $k$. A vector bundle $E$ on $Y$ is called exceptional if $\operatorname{Hom}(E, E) \simeq k$ and $\operatorname{Ext}^{i}(E, E)=0$, for all $i \geq 1$.

Exceptional bundles were introduced by Drézet and Le Potier [6 in order to characterise the Chern classes of stable coherent sheaves on $\mathbb{P}^{2}$. The set of exceptional bundles on the projective plane and, more generally, on projective spaces was afterwards studied by Drézet, Gorodentsev and Rudakov in [5, 8 and 12. For general results about exceptional bundles on projective varieties, see, for example, [2], 3], 4] or [10].

The study of exceptional vector bundles plays an important role for the study of stable vector bundles and therefore, in the classification of vector bundles on a projective variety. A long-standing problem in Algebraic Geometry is to determine the stability of exceptional bundles on smooth projective varieties. Few contributions to this very difficult and interesting problem have been made. In 6], Drézet and Le Potier prove that any exceptional bundle on $\mathbb{P}^{2}$ is stable. The stability of exceptional bundles on the quadric $\mathbb{P}^{1} \times \mathbb{P}^{1}$ is proved by Gorodentsev in [7, and the stability of exceptional Steiner bundles on $\mathbb{P}^{n}, n \geq 2$, and on the hyperquadric $Q_{n} \subset \mathbb{P}^{n+1}, n \geq 3$, is proved in [1] and 13, respectively. In [14, Zube proves that any exceptional bundle on $K 3$ surfaces with Picard group $\mathbb{Z}$ is stable and uses this result to prove that exceptional bundles on $\mathbb{P}^{3}$ are stable. His main idea is to prove that given an exceptional bundle $E$ on $\mathbb{P}^{3}$ its restriction $\left.E\right|_{X}$ to a $K 3$ surface $X$

Received by the editors January 29, 2007, and, in revised form, February 23, 2007.

2000 Mathematics Subject Classification. Primary 14F05.

Key words and phrases. Exceptional vector bundles, stability.

The first author was partially supported by MTM2004-00666.

The second author was partially supported by Fundação para a Ciência e Tecnologia under grant SFRH/BD/16589/2004, and by Departamento de Métodos Quantitativos do Instituto Superior de Ciências do Trabalho e da Empresa. 
with $\operatorname{Pic}(X) \simeq \mathbb{Z}$ is an exceptional vector bundle on $X$ and hence it is stable with respect to any ample divisor on $X$.

The work of Zube provided the inspiration for the present paper, in which we prove that any exceptional bundle on a smooth complete intersection 3-fold $Y \subset \mathbb{P}^{n}$ of type $\left(d_{1}, \ldots, d_{n-3}\right)$ with $d_{1}+\cdots+d_{n-3} \leq n$ and $n \geq 4$, is stable.

It is worth remarking that in most of the literature an exceptional bundle $E$ on a $K 3$ surface $X$ is by definition a simple and rigid vector bundle, that is, a vector bundle satisfying $\operatorname{Hom}(E, E) \simeq k$ and $\operatorname{Ext}^{1}(E, E)=0$. Due to Serre duality it follows that $\operatorname{Ext}^{2}(E, E) \simeq k$. In order to avoid any confusion with the definition of exceptional bundles given above, in what follows we will call them simple and rigid bundles.

The paper is organised as follows. Section 1 contains the basic concepts and properties on sheaves, stability and $K 3$ surfaces and that which is essential for the following section. We use the definition of stability of Mumford and Takemoto. In section 2, we develop the main tools in order to reach our goal. Namely, we prove that if $Y \subset \mathbb{P}^{n}, n \geq 4$, is a smooth complete intersection variety of dimension $m \geq 3$ and of type $\left(d_{1}, \ldots, d_{n-m}\right)$ with $d_{1}+\cdots+d_{n-m} \leq n-m+3$, then $Y$ contains a $K 3$ surface $X$ (Proposition 2.1). We then restrict our attention to the case $m=3$ and prove that an exceptional bundle on $Y$, when restricted to $X$, is a simple and rigid vector bundle on $X$ (Proposition 2.2). By [11, Theorem 7.5], choosing $X$ general, the Picard group of $X$ is $\mathbb{Z}$ and thus, Zube's result implies that $\left.E\right|_{X}$ is stable with respect to any ample divisor $H$. This will allow us to prove our main result, which asserts the following:

Theorem 0.1. Let $E$ be an exceptional vector bundle on a smooth complete intersection 3 -fold $Y \subset \mathbb{P}^{n}$ of type $\left(d_{1}, \ldots, d_{n-3}\right)$ with $d_{1}+\cdots+d_{n-3} \leq n$ and $n \geq 4$. Then $E$ is stable with respect to any ample divisor $H$.

Remark 0.2. It is worthwhile to point out that by Lefschetz's theorem $Y$ has a unique polarisation.

\section{Preliminaries}

In this section we introduce the main definitions and results needed in the sequel and set up some notation. We start with some basic concepts on sheaves.

Throughout this paper all projective varieties are defined over an algebraically closed field $k$. Given any sheaf $E$ on a smooth projective variety, we will denote by $E^{\vee}$ its dual. For any two sheaves $E$ and $F$ on $X, h^{i}(E, F)$ will denote $\operatorname{dim} \operatorname{Ext}^{i}(E, F)$.

Definition 1.1. Let $X$ be a smooth projective variety. A coherent sheaf $E$ on $X$ is simple if

$$
\operatorname{Hom}(E, E) \simeq k .
$$

The sheaf $E$ is rigid if $\operatorname{Ext}^{1}(E, E)=0$.

If $E$ is simple and, furthermore, it satisfies

$$
\operatorname{Ext}^{i}(E, E)=0 \text {, for all } i \geq 1,
$$

then $E$ is exceptional. 


\section{Examples 1.2.}

(1) Instanton bundles on $\mathbb{P}^{2 n+1}$ are simple but not rigid and thus not exceptional.

(2) Let $X$ be a $K 3$ surface in $\mathbb{P}^{3}$, that is, a hypersurface of degree 4 . If $E$ is a simple and rigid vector bundle on $X$, then $E$ is not exceptional, since $H^{2}\left(E \otimes E^{\vee}\right) \simeq H^{0}\left(E \otimes E^{\vee}\right) \simeq k$.

(3) The line bundles $\mathcal{O}_{\mathbb{P}^{n}}(l), l \in \mathbb{Z}$, and the tangent bundle $T_{\mathbb{P}^{n}}$ on $\mathbb{P}^{n}$ are exceptional bundles on $\mathbb{P}^{n}$. In particular, they are simple and rigid.

Definition 1.3. Let $F$ be a coherent sheaf on a smooth projective variety. If the natural map $F \longrightarrow F^{\vee \vee}$ of $F$ to its double dual is an isomorphism, then $F$ is reflexive.

For example, locally free sheaves are reflexive. We will need the following two results on reflexive sheaves, whose proof can be found in Hartshorne's paper 9 , section 1].

Proposition 1.4. Let $X$ be a smooth algebraic variety. If $F$ is a reflexive sheaf on $X$, then $F$ is locally free except along a closed subset $Y$ of codimension $\geq 3$. In particular, if $X$ is a 2-dimensional variety, then $F$ is locally free.

Proposition 1.5. Let $X$ be a smooth algebraic variety. A coherent sheaf $F$ on $X$ is reflexive if and only if (at least locally) it can be included in an exact sequence

$$
0 \longrightarrow F \longrightarrow E \longrightarrow G \longrightarrow 0,
$$

where $E$ is locally free and $G$ is torsion-free.

Let $X$ be a smooth projective variety of dimension $n$ and let $E$ be a torsion-free coherent sheaf of rank $r$ on $X$. Recall that the slope of $E$ is the rational number

$$
\mu_{H}(E)=\frac{c_{1} H^{n-1}}{r}
$$

where $H$ is a fixed ample line bundle on $X$ and $c_{1}$ is the first Chern class of $E$.

Definition 1.6 (Mumford/Takemoto). A torsion-free coherent sheaf $E$ on a smooth projective variety $X$ is semi-stable with respect to $H$ if $\mu_{H}(F) \leq \mu_{H}(E)$, for every coherent subsheaf $F \subset E$, with $0<\operatorname{rank} F<\operatorname{rank} E$. If, moreover, $\mu_{H}(F)<\mu_{H}(E)$, then $E$ is stable.

Remark 1.7. The stability of a torsion-free sheaf $E$ can be proved if we consider only subsheaves $F$ of $E$ such that the quotient $E / F$ is torsion-free. In fact, if $E$ is a torsion-free sheaf on a smooth projective variety $X, E$ is stable (resp. semi-stable) if and only if $\mu_{H}(F)<\mu_{H}(E)$ (resp. $\mu_{H}(F) \leq \mu_{H}(E)$ ), for all coherent subsheaves $F \subset E$ with $0<\operatorname{rank} F<\operatorname{rank} E$ whose quotient is torsion-free.

One of the fundamental tools for our purposes will be to reduce our study to $K 3$ surfaces. Let us give their definition and some examples.

If $X$ is a smooth algebraic surface, we denote by $K_{X}$ the canonical line bundle on $X$. Recall that $h^{1}\left(X, \mathcal{O}_{X}\right)$ is called the irregularity of $X$.

Definition 1.8. A $K 3$ surface $X$ over an algebraically closed field $k$ is a smooth algebraic surface with trivial canonical line bundle, $K_{X} \simeq \mathcal{O}_{X}$, and irregularity $h^{1}\left(X, \mathcal{O}_{X}\right)=0$. 
It follows from Serre duality that for any pair of coherent sheaves $E$ and $F$ on a $K 3$ surface $X$,

$$
\operatorname{Ext}^{i}(E, F)^{*} \simeq \operatorname{Ext}^{2-i}(F, E) .
$$

The following are some of the most common examples of $K 3$ surfaces.

\section{Examples 1.9.}

(1) A smooth hypersurface of degree 4 in $\mathbb{P}^{3}$.

(2) A smooth complete intersection of a quadric and a cubic hypersurface in $\mathbb{P}^{4}$.

(3) A smooth complete intersection of three quadrics in $\mathbb{P}^{5}$.

(4) Kummer surfaces.

The next theorem, proved by Zube [14, will play an essential role in our work.

Theorem 1.10. Let $E$ be a simple and rigid bundle on a $K 3$ surface $X$ with Picard group $\mathbb{Z}$. Then $E$ is stable with respect to any ample divisor $H$.

\section{Stable exceptional bundles on hypersurfaces in $\mathbb{P}^{4}$}

In this section we prove the stability of exceptional vector bundles on a smooth complete intersection 3-fold $Y \subset \mathbb{P}^{n}$ of type $\left(d_{1}, \ldots, d_{n-3}\right)$ with $d_{1}+\cdots+d_{n-3} \leq n$ and $n \geq 4$. Our first goal is to prove that there exists a $K 3$ surface $X$ contained in $Y$ for which the restriction of an exceptional vector bundle $E$ on $Y$ to $X$ is a simple and rigid vector bundle on $X$.

The next proposition guarantees that inside a smooth complete intersection 3fold $Y \subset \mathbb{P}^{n}$ of type $\left(d_{1}, \ldots, d_{n-3}\right)$ with $d_{1}+\cdots+d_{n-3} \leq n$ and $n \geq 4$ there always exists a $K 3$ surface. It also provides us more examples of $K 3$ surfaces on $\mathbb{P}^{n}$.

Proposition 2.1. Let $Y \subset \mathbb{P}^{n}, n \geq 4$, be a smooth complete intersection of dimension $m \geq 3$ and type $\left(d_{1}, \ldots, d_{n-m}\right)$. Assume that $d_{1}+\cdots+d_{n-m} \leq n-m+3$. Then $Y$ contains a $K 3$ surface.

Proof. Let $Y=\mathbb{V}\left(f_{1}, \ldots, f_{n-m}\right) \subset \mathbb{P}^{n}, n \geq 4$, where $f_{i} \in K\left[x_{0}, \ldots, x_{n}\right]$ is a nonzero form of degree $d_{i}, 1 \leq i \leq n-m$.

Define $X:=\mathbb{V}\left(f_{1}, \ldots, f_{n-m}, g_{1}, \ldots, g_{m-2}\right) \subset Y$, where $g_{i}, i=1, \ldots, m-2$, are general forms of degree $e_{i} \geq 1$ and such that $\sum_{i=1}^{m-2} e_{i}=n+1-\sum_{i=1}^{n-m} d_{i}$. Then $X$ is a complete intersection surface and thus

$$
K_{X}=\mathcal{O}_{X}\left(\sum_{i=1}^{m-2} e_{i}+\sum_{i=1}^{n-m} d_{i}-n-1\right)=\mathcal{O}_{X}
$$

and $H^{1}\left(X, \mathcal{O}_{X}\right)=0$.

Therefore, $X$ is a $K 3$ surface contained in the hypersurface $Y$.

Let $Y=\mathbb{V}\left(f_{1}, \ldots, f_{n-3}\right) \subset \mathbb{P}^{n}, n \geq 4$, be a smooth complete intersection 3-fold of type $\left(d_{1}, \ldots, d_{n-3}\right)$ with $d_{1}+\cdots+d_{n-3} \leq n$. By Proposition 2.1 .

$$
X:=\mathbb{V}\left(f_{1}, \ldots, f_{n-3}, g\right),
$$

where $g$ is a nonzero form of degree $n+1-\sum_{i=1}^{n-3} d_{i}$ in a $K 3$ surface contained in $Y$.

Let $E$ be an exceptional vector bundle on $Y$. Since $E$ is a locally free sheaf, the restriction $\left.E\right|_{X}$ is also a locally free sheaf, i.e., is a vector bundle on $X$. Now we prove that the vector bundle $\left.E\right|_{X}$ is simple and rigid. 
Proposition 2.2. Let $E$ be an exceptional vector bundle on a smooth complete intersection 3-fold $Y=\mathbb{V}\left(f_{1}, \ldots, f_{n-3}\right) \subset \mathbb{P}^{n}, n \geq 4$, of type $\left(d_{1}, \ldots, d_{n-3}\right)$ with $d_{1}+\cdots+d_{n-3} \leq n$, and let $X$ be a K3 surface as in (2.1) contained in $Y$. Then the vector bundle $\left.E\right|_{X}$ on $X$ is simple and rigid.

Proof. Consider the short exact sequence

$$
0 \longrightarrow \mathcal{O}_{Y}\left(\sum_{i=1}^{n-3} d_{i}-n-1\right) \longrightarrow \mathcal{O}_{Y} \longrightarrow \mathcal{O}_{X} \longrightarrow 0 .
$$

Tensoring it by $E^{\vee} \otimes E$ and taking cohomology, we get

$$
\begin{array}{r}
0 \longrightarrow H^{0}\left(E^{\vee} \otimes E\left(\sum_{i=1}^{n-3} d_{i}-n-1\right)\right) \longrightarrow H^{0}\left(E^{\vee} \otimes E\right) \longrightarrow H^{0}\left(\left.\left(E^{\vee} \otimes E\right)\right|_{X}\right) \\
\longrightarrow H^{1}\left(E^{\vee} \otimes E\left(\sum_{i=1}^{n-3} d_{i}-n-1\right)\right) \longrightarrow H^{1}\left(E^{\vee} \otimes E\right) \longrightarrow H^{1}\left(\left.\left(E^{\vee} \otimes E\right)\right|_{X}\right) \\
\longrightarrow H^{2}\left(E^{\vee} \otimes E\left(\sum_{i=1}^{n-3} d_{i}-n-1\right)\right) \longrightarrow H^{2}\left(E^{\vee} \otimes E\right) \longrightarrow H^{2}\left(\left.\left(E^{\vee} \otimes E\right)\right|_{X}\right) \longrightarrow \cdots
\end{array}
$$

Since $E$ is exceptional on $Y, H^{0}\left(E^{\vee} \otimes E\right) \simeq k$ and $H^{i}\left(E^{\vee} \otimes E\right)=0$ for all $i>0$. Moreover, the canonical bundle of $Y$ is $K_{Y} \simeq \mathcal{O}_{Y}\left(\sum_{i=1}^{n-3} d_{i}-n-1\right)$. Hence, by Serre duality we have

$$
\begin{aligned}
& H^{0}\left(E^{\vee} \otimes E\left(\sum_{i=1}^{n-3} d_{i}-n-1\right)\right) \simeq H^{3}\left(E^{\vee} \otimes E\right)^{*}=0, \\
& H^{1}\left(E^{\vee} \otimes E\left(\sum_{i=1}^{n-3} d_{i}-n-1\right)\right) \simeq H^{2}\left(E^{\vee} \otimes E\right)^{*}=0, \\
& H^{2}\left(E^{\vee} \otimes E\left(\sum_{i=1}^{n-3} d_{i}-n-1\right)\right) \simeq H^{1}\left(E^{\vee} \otimes E\right)^{*}=0,
\end{aligned}
$$

and so we deduce that

$$
H^{0}\left(\left.\left(E^{\vee} \otimes E\right)\right|_{X}\right) \simeq H^{0}\left(E^{\vee} \otimes E\right) \simeq k, \quad H^{1}\left(\left.\left(E^{\vee} \otimes E\right)\right|_{X}\right)=0 .
$$

Therefore, $\left.E\right|_{X}$ is simple and rigid.

To apply Zube's theorem we need to guarantee that $\operatorname{Pic}(X) \cong \mathbb{Z}$. To this end, we will apply Moišezon's theorem.

Theorem 2.3. Let $Y \subset \mathbb{P}^{n}$ be a smooth irreducible 3-fold and let $X \subset Y \subset \mathbb{P}^{n}$ be the surface cut out by a general form of degree $d$. If $h^{2}\left(Y, \mathcal{O}_{Y}\right)<h^{2}\left(X, \mathcal{O}_{X}\right)$, then $\operatorname{Pic}(Y) \simeq \operatorname{Pic}(X)$.

Proof. See [11, Theorem 7.5].

At this point, we conclude that if $E$ is an exceptional vector bundle on a smooth complete intersection 3-fold $Y \subset \mathbb{P}^{n}$ of type $\left(d_{1}, \ldots, d_{n-3}\right)$ with $d_{1}+\cdots+d_{n-3} \leq n$ and $n \geq 4$, then the restriction $\left.E\right|_{X}$ to a $K 3$ surface $X$ defined as in (2.1) is stable provided that $g$ is general. In fact, by Lefschetz's theorem, $\operatorname{Pic}(Y) \simeq \mathbb{Z}$ and it is easy to check that under our numerical assumptions we have $h^{2}\left(Y, \mathcal{O}_{Y}\right)<h^{2}\left(X, \mathcal{O}_{X}\right)$. Therefore, Moišezon's theorem applies, and we conclude that $\operatorname{Pic}(X) \simeq \mathbb{Z}$ and thus Zube's result, Theorem 1.10, implies that $\left.E\right|_{X}$ is stable. 
We can now prove our main theorem.

Theorem 2.4. Let $E$ be an exceptional vector bundle on a smooth complete intersection 3 -fold $Y \subset \mathbb{P}^{n}$ of type $\left(d_{1}, \ldots, d_{n-3}\right)$ with $d_{1}+\cdots+d_{n-3} \leq n$ and $n \geq 4$. Then $E$ is stable with respect to any ample divisor $H$.

Proof. Suppose $E$ is not stable. Then there exists a subsheaf $F$ of $E$ whose quotient is torsion-free and such that $\mu_{H}(E) \leq \mu_{H}(F)$ (see Remark 1.7). In particular, $F$ can be included in a short exact sequence

$$
0 \longrightarrow F \longrightarrow E \longrightarrow G \longrightarrow 0
$$

where $E$ is locally free and $G$ is torsion-free. Therefore, by Proposition 1.5, $F$ is reflexive and hence, by Proposition 1.4. $F$ is locally free outside finitely many points.

Let $f_{1}, \ldots, f_{n-3}$ be the nonzero forms of degree $d_{i}, 1 \leq i \leq n-3$, defining $Y$ and let $X=\mathbb{V}\left(f_{1}, \ldots, f_{n-3}, g\right) \subset Y$ be a $K 3$ surface contained in $Y$, where $g$ is a nonzero form of degree $n+1-\sum_{i=1}^{n-3} d_{i}$ (see the proof of Proposition 2.1). As mentioned above, if we choose $g$ general, then Molšezon's theorem allows us to conclude that the Picard group of $X$ is $\mathbb{Z}$. Moreover, we may assume that $X$ does not contain the points where $F$ might not be locally free. Hence the restriction $\left.F\right|_{X}$ is a locally free sheaf, and so we may assume that $\left.F\right|_{X}$ is a subbundle of $\left.E\right|_{X}$.

On the other hand, Proposition 2.2 implies that $\left.E\right|_{X}$ is a simple and rigid vector bundle on $X$. Applying Theorem 1.10, $\left.E\right|_{X}$ is stable with respect to any ample divisor $H$ and thus

$$
\mu_{H}\left(\left.F\right|_{X}\right)=\frac{c_{1}(F)}{\operatorname{rk}(F)}=\mu_{H}(F)<\mu_{H}(E)=\frac{c_{1}(E)}{\operatorname{rk}(E)}=\mu_{H}\left(\left.E\right|_{X}\right),
$$

contradicting our hypothesis that $E$ is not stable.

Remark 2.5. If we think of doing a similar study for exceptional bundles on $\mathbb{P}^{n}$ for $n \geq 5$ (resp. in $Y \subset \mathbb{P}^{n}, n \geq 4$, a smooth complete intersection of dimension $m>3$ and of type $\left(d_{1}, \ldots, d_{n-m}\right)$ and such that $\left.d_{1}+\cdots+d_{n-m} \leq n-m+3\right)$, we see that by Proposition 2.1] we can also find a $K 3$ surface $X$ in $\mathbb{P}^{n}$ (resp. in $\left.Y \subset \mathbb{P}^{n}\right)$. However, the problem is that we cannot guarantee that $\left.E\right|_{X}$ is simple and rigid, as we did in Proposition 2.2 for the case $m=3$.

As an immediate consequence of Theorem 2.4 we can state the following:

Corollary 2.6. Let $E$ be an exceptional vector bundle on a smooth hypersurface $Y \subset \mathbb{P}^{4}$ of degree $d, 1 \leq d \leq 4$. Then $E$ is stable with respect to any ample divisor $H$.

In particular, any exceptional bundle $E$ on the smooth hyperquadric $Q_{3} \subset \mathbb{P}^{4}$ is stable with respect to any ample divisor $H$.

Remark 2.7. $\operatorname{Ext}^{1}(E, E)$ represents the local deformation functor. We have that exceptional bundles on a smooth complete intersection 3 -fold $Y \subset \mathbb{P}^{n}$ of type $\left(d_{1}, \ldots, d_{n-3}\right)$ with $d_{1}+\cdots+d_{n-3} \leq n$ and $n \geq 4$ are infinitesimal rigid. So we deduce that the moduli space of exceptional bundles on smooth complete intersection 3-folds $Y \subset \mathbb{P}^{n}$ of type $\left(d_{1}, \ldots, d_{n-3}\right)$ with $d_{1}+\cdots+d_{n-3} \leq n$ and $n \geq 4$ is a discrete set of points. 


\section{ACKNOWLEDGEMENT}

We would like to thank Laura Costa for her very helpful comments during the preparation of this work.

\section{REFERENCES}

1. Brambilla, C., Cokernel bundles and Fibonacci bundles, Math. Nachr. (to appear).

2. Costa, L., Miró-Roig, R. M., Tilting sheaves on toric varieties, Math. Z. 248 (2004), 849-865. MR2103545 (2005m:14023)

3. Costa, L., Miró-Roig, R. M., Derived categories of projective bundles, Proc. Amer. Math. Soc. 133, 9 (2005), 2533-2537. MR2146195 (2006d:14017)

4. Costa, L., Miró-Roig, R. M., Geometric collections and Castelnuovo-Mumford regularity, Math. Proc. Cambridge Philos. Soc. 143 (2007), 557-578.

5. Drézet, J.-M., Fibrés exceptionnels et suite spectrale de Beilinson généralisée sur $\mathbb{P}_{2}(\mathbb{C})$, Math. Ann. 275 (1986), 25-48. MR849052 (88b:14014)

6. Drézet, J.-M., Le Potier, J., Fibrés stables et fibrés exceptionnels sur $\mathbb{P}_{2}$, Ann. Sci. École Norm. Sup. 18 (1985), 2, 193-243. MR816365 (87e:14014)

7. Gorodentsev, A. L., Exceptional bundles on surfaces with a moving anticanonical class, Math. USSR Isv. 33 (1989), 67-83. MR966982 (90d:14017)

8. Gorodentsev, A. L., Rudakov, A. N., Exceptional vector bundles on projective spaces, Duke Math. J. 54 (1987), 115-130. MR885779 (88e:14018)

9. Hartshorne, R., Stable reflexive sheaves, Math. Ann. 254 (1980), 121-176. MR597077 (82b:14011)

10. Kapranov, M. M., On the derived categories of coherent sheaves on some homogeneous spaces, Invent. Math. 92 (1988), 479-508. MR939472 (89g:18018)

11. Moišezon, B. G., Algebraic homology classes on algebraic varieties, Math. USSR Izv. 1 (1967), 209-251. MR0213351(35:4215)

12. Rudakov, A. N., Markov numbers and exceptional bundles on $\mathbb{P}^{2}$, Izv. Akad. Nauk SSSR Ser. Mat. 52 (1988), 100-112; English transl. in Math. USSR Izv. 32 (1989). MR936525|(89f:14012)

13. Soares, H., Steiner bundles on the hyperquadric $Q_{n} \subset \mathbb{P}^{n+1}, n \geq 3$, Comm. Algebra 35 (2007), 4116-4136. MR2372324

14. Zube, D. Yu., The stability of exceptional bundles on three-dimensional projective space, in Helices and vector bundles, London Math. Soc. Lecture Notes Series, vol. 148 (1990), Cambridge Univ. Press, Cambridge. MR1074787 (91j:14038)

Facultat de Matemátiques, Departament d’Àlgebra i Geometria, Universitat de Barcelona, Gran Via de les Corts Catalanes 585, 08007 Barcelona, Spain

E-mail address: miro@ub.edu

ISCTE Business School, Departamento de Métodos Quantitativos, Edifício iscte, Av. Forças Armadas, 1649-026 Lisboa, Portugal

E-mail address: helena.soares@ub.edu 